Georgian Mathematical Journal

Volume 11 (2004), Number 1, 83-92

\title{
COMMON FIXED POINTS ITERATION PROCESSES FOR A FINITE FAMILY OF ASYMPTOTICALLY NONEXPANSIVE MAPPINGS
}

\author{
JUI-CHI HUANG
}

\begin{abstract}
Let $E$ be a uniformly convex Banach space which satisfies Opial's condition or its dual $E^{*}$ has the Kadec-Klee property, $C$ a nonempty closed convex subset of $E$, and $T_{j}: C \rightarrow C$ an asymptotically nonexpansive mapping for each $j=1,2, \ldots, r$. Suppose $\left\{x_{n}\right\}$ is generated iteratively by

$x_{0} \in C, \quad x_{n+1}=\left(1-\alpha_{n(r)}\right) x_{n}+\alpha_{n(r)} \frac{1}{n+1} \sum_{i=0}^{n} T_{r}^{i} U_{n(r-1)} x_{n}, \quad n=0,1,2, \ldots$, where $U_{n(j)}=\left(1-\alpha_{n(j)}\right) I+\alpha_{n(j)} \frac{1}{n+1} \sum_{i=0}^{n} T_{j}^{i} U_{n(j-1)}, j=1,2, \ldots, r, U_{n(0)}=$ $I, I$ is the identity map and $\left\{\alpha_{n(j)}\right\}$ is a suitable sequence in $[0,1]$. If the set $\cap_{j=1}^{r} F\left(T_{j}\right)$ of common fixed points of $\left\{T_{j}\right\}_{j=1}^{r}$ is nonempty, then weak convergence of $\left\{x_{n}\right\}$ to some $p \in \cap_{j=1}^{r} F\left(T_{j}\right)$ is obtained.
\end{abstract}

2000 Mathematics Subject Classification: 47H10.

Key words and phrases: Asymptotically nonexpansive mapping, Fixed point, uniformly convex Banach space, Opial's condition, Kadec-Klee property.

\section{INTRODUCTION}

Let $C$ be a nonempty closed convex subset of a real Banach space $E$ and let $T$ be a mapping of $C$ into itself. Then, we denote by $F(T)$ the set of fixed points of $T$. A mapping $T$ of $C$ into itself is said to be nonexpansive if

$$
\|T x-T y\| \leq\|x-y\|
$$

for all $x, y \in C$ and a mapping $T$ of $C$ into itself is said to be asymptotically nonexpansive with Lipschitz constants $\left\{k_{n}\right\}$ if $\overline{\lim }_{n \rightarrow \infty} k_{n} \leq 1$ and

$$
\left\|T^{n} x-T^{n} y\right\| \leq k_{n}\|x-y\|
$$

for all $x, y \in C$ and $n=0,1,2, \ldots$

In 1998, Atsushiba and Takahashi [1] introduced an iteration procedure of Mann's type for approximating common fixed points of two nonexpansive mappings $S$ and $T$ as follows:

$$
x_{0} \in C, \quad x_{n+1}=\left(1-\alpha_{n}\right) x_{n}+\alpha_{n} \frac{1}{(n+1)^{2}} \sum_{i, j=0}^{n} S^{i} T^{j} x_{n}, \quad n=0,1,2, \ldots,
$$

where $\left\{\alpha_{n}\right\}_{n=0}^{\infty}$ is a sequence in $[0,1]$. More precisely, they proved the following theorem: 
Theorem A (see [1, Theorem 1]). Let E be a uniformly convex Banach space which satisfies Opial's condition or whose norm is Fréchet differentiable. Let $C$ be a nonempty closed convex subset of $E$. Let $S$ and $T$ be nonexpansive mappings of $C$ into itself such that $S T=T S$ and $F(S) \cap F(T) \neq \varnothing$. Suppose that $x_{0} \in C$ and $\left\{x_{n}\right\}_{n=0}^{\infty}$ is given by (1.3). If $\left\{\alpha_{n}\right\}_{n=0}^{\infty}$ is chosen so that $0<a \leq \alpha_{n} \leq 1$ for some constant $a$, then $\left\{x_{n}\right\}_{n=0}^{\infty}$ converges weakly to a common fixed point of $S$ and $T$.

Let $C$ be a nonempty convex subset of a Banach space $E$. Let $T_{j}: C \rightarrow C$ be a given mapping for each $j=1,2, \ldots, r$. In this paper, we consider the following iteration scheme generated by $T_{1}, T_{2}, \ldots, T_{r}$ :

$$
\begin{aligned}
U_{n(1)} & =\left(1-\alpha_{n(1)}\right) I+\alpha_{n(1)} \frac{1}{n+1} \sum_{i=0}^{n} T_{1}^{i} U_{n(0)}, \\
U_{n(2)} & =\left(1-\alpha_{n(2)}\right) I+\alpha_{n(2)} \frac{1}{n+1} \sum_{i=0}^{n} T_{2}^{i} U_{n(1)}, \\
\vdots & \\
U_{n(r)} & =\left(1-\alpha_{n(r)}\right) I+\alpha_{n(r)} \frac{1}{n+1} \sum_{i=0}^{n} T_{r}^{i} U_{n(r-1)}, \\
x_{0} \in C, x_{n+1} & =\left(1-\alpha_{n(r)}\right) x_{n}+\alpha_{n(r)} \frac{1}{n+1} \sum_{i=0}^{n} T_{r}^{i} U_{n(r-1)} x_{n}, \quad n=0,1,2, \ldots,
\end{aligned}
$$

where $T_{j}^{0}=U_{n(0)}:=I, I$ is the identity map, and $\left\{\alpha_{n(j)}\right\}_{n=0}^{\infty}$ is a sequence in $[0,1]$ for each $j=1,2, \ldots, r$. Using this scheme we improve Theorem A by removing the assumption that $S T=T S$ and extending the two nonexpansiveness mappings to a finite family of asymptotically nonexpansive mappings. Since the duals of reflexive Banach spaces with a Fréchet differentiable norm have the Kadec-Klee property (see [4] or [5]), we consider our main theorems under the assumption that $E$ is a uniformly convex Banach space such that its dual $E^{*}$ has the Kadec-Klee property.

\section{Preliminaries}

Throughout this paper, $E$ is a real Banach space and $E^{*}$ is the dual space of $E$. We use the notation $\overline{\lim }=\lim \sup$ and $\underline{\lim }=\lim \inf$, denote by $\mathbb{N}$ the set of all nonnegative integers, denote $\max \{a, 0\}$ by $(a)_{+}$for a real number $a$, and put $B_{d}=\{x \in E:\|x\| \leq d\}$ for $d>0$.

A Banach space $E$ is said to be uniformly convex if the modulus of convexity of $E$

$$
\delta_{E}(\varepsilon)=\inf \left\{1-\frac{1}{2}\|x+y\|:\|x\| \leq 1,\|y\| \leq 1,\|x-y\| \geq \varepsilon\right\}>0
$$

for all $0<\varepsilon \leq 2$. It is well-known that a uniformly convex Banach space is reflexive. We say that $E$ satisfies Opial's condition [9] if for each sequence $\left\{x_{n}\right\}$ 
of $E$ converging weakly to $x, x \neq y$ implies

$$
\varliminf_{n \rightarrow \infty}\left\|x_{n}-x\right\|<\varliminf_{n \rightarrow \infty}\left\|x_{n}-y\right\|,
$$

and $E$ is said to have the Kadec-Klee property (KK-property) [8] if whenever $x_{n} \rightarrow x$ weakly with $\left\|x_{n}\right\| \rightarrow\|x\|$, it follows that $x_{n} \rightarrow x$ strongly. The norm $\|$.$\| of E$ is said to be Fréchet differentiable if for all $x \in S(E)$, the limit

$$
\lim _{t \rightarrow 0} \frac{\|x+t y\|-\|x\|}{t}
$$

exists uniformly for all $y \in S(E)$ where $S(E)=\{x \in E:\|x\|=1\}$. Note that duals of reflexive Banach spaces with Fréchet differentiable norms have the KK-property (see [4] or [5]) and there exist uniformly convex Banach spaces which have neither a Fréchet differentiable norm nor the Opial property but their duals do have the KK-property (see [6, Example 3.1]).

In the sequel we shall need the following lemmas.

Lemma 2.1 (see [3, Lemma 3]). Let $\left\{\psi_{n}\right\}_{n=0}^{\infty}$ and $\left\{\varphi_{n}\right\}_{n=0}^{\infty}$ be sequences of nonnegative real numbers satisfying the inequality

$$
\psi_{n+1} \leq\left(1+\varphi_{n}\right) \psi_{n}, \quad n=0,1,2, \ldots .
$$

If $\sum_{n=0}^{\infty} \varphi_{n}<\infty$, then $\lim _{n \rightarrow \infty} \psi_{n}$ exists.

Lemma 2.2 (see [10, Lemma 3]). Let $C$ be a nonempty closed convex subset of a uniformly convex Banach space $E$. Let $T$ be an asymptotically nonexpansive mapping from $C$ into itself such that $F(T)$ is nonempty. Then for each $r>0$, there holds

$$
\varlimsup_{m \rightarrow \infty} \varlimsup_{n \rightarrow \infty} \sup _{x \in C \cap B_{d}}\left\|\frac{1}{n+1} \sum_{i=0}^{n} T^{i} x-T^{m}\left(\frac{1}{n+1} \sum_{i=0}^{n} T^{i} x\right)\right\|=0 .
$$

Lemma 2.3 (see [6, Lemma 3.2]). Let E be a uniformly convex Banach space such that its dual $E^{*}$ has the KK-property. Suppose that $\left\{x_{n}\right\}_{n=0}^{\infty}$ is a bounded sequence such that $\lim _{n \rightarrow \infty}\left\|t x_{n}+(1-t) f_{1}-f_{2}\right\|$ exists for all $t \in[0,1]$ and $f_{1}$, $f_{2} \in \omega_{w}\left(x_{n}\right)$. Then $\omega_{w}\left(x_{n}\right)$ is a singleton. Here, $\omega_{w}\left(x_{n}\right)$ denotes the set of weak subsequential limits of $\left\{x_{n}\right\}$.

Lemma 2.4 (see [2, Lemma 1.1]). Let E be a uniformly convex Banach space, $K$ be a nonempty bounded closed convex subset of $E$. Then there exists a strictly increasing, continuous and convex function $g:[0, \infty) \rightarrow[0, \infty)$ with $g(0)=0$ such that, for any Lipschitzian continuous mapping $V: K \rightarrow E, x$, $y \in K$ and $t \in[0,1]$, the following inequality holds

$$
\|V(t x+(1-t) y)-(t V x+(1-t) V y)\| \leq L g^{-1}\left(\|x-y\|-L^{-1}\|V x-V y\|\right),
$$

where $L \geq 0$ is the Lipschitz constant of $V$.

Lemma 2.5 (see [13, Theorem 2]). Let E be a uniformly convex Banach space and $d>0$. Then there exists a continuous, strictly increasing and convex function $g:[0, \infty) \rightarrow[0, \infty)$ such that $g(0)=0$ and

$$
\|\lambda x+(1-\lambda) y\|^{2} \leq \lambda\|x\|^{2}+(1-\lambda)\|y\|^{2}-\lambda(1-\lambda) g(\|x-y\|)
$$


for all $x, y \in B_{d}$ and $\lambda \in[0,1]$.

\section{Main Results}

For our main results, we need the following lemmas.

Lemma 3.1. Let $C$ be a nonempty closed convex subset of a uniformly convex Banach space $E$ and $S_{n}: C \rightarrow C$ be Lipschitzian mapping with the Lipschitz constant $L_{n} \geq 1$ such that $\sum_{n=0}^{\infty}\left(L_{n}-1\right)<\infty$ and $\cap_{n=0}^{\infty} F\left(S_{n}\right) \neq \varnothing$. Suppose that $\left\{x_{n}\right\}$ is given by $x_{n+1}=S_{n} x_{n}$ for all $n \in \mathbb{N}$. Then $\lim _{n \rightarrow \infty} \| t x_{n}+(1-$ t) $f_{1}-f_{2} \|$ exists for all $f_{1}, f_{2} \in \cap_{n=0}^{\infty} F\left(S_{n}\right)$ and $t \in[0,1]$.

Proof. This is basically the proof of Lemma 2.2 of Tan and $\mathrm{Xu}$ [12]. For completeness and without the assumption that $C$ is bounded, we sketch the details. By Lemma 2.1 of Tan and $\mathrm{Xu}[12]$, we know that $\lim _{n \rightarrow \infty}\left\|x_{n}-f\right\|$ exists for each $f \in \cap_{n=0}^{\infty} F\left(S_{n}\right)$. Therefore, there exists $d>0$ such that $\sup _{n \in \mathbb{N}}\left\{\left\|x_{n}-f_{1}\right\|\right\} \leq d$. Take $K=\left\{x \in C:\left\|x-f_{1}\right\| \leq d\right\}$. Then $K$ is a nonempty bounded closed convex subset of $E$. Set

$$
V_{n, m}=S_{n+m-1} S_{n+m-2} \cdots S_{n}
$$

for all $n, m \geq 0$. Then $V_{n, m} x_{n}=x_{n+m}$ and for all $x, y \in C$

$$
\left\|V_{n, m} x-V_{n, m} y\right\| \leq M_{n}\|x-y\|
$$

where $M_{n}=\prod_{j=n}^{\infty} L_{j}$. Since $\sum_{n=0}^{\infty}\left(L_{n}-1\right)<\infty$, we have $M_{n} \rightarrow 1$ as $n \rightarrow \infty$. Setting

$$
b_{n, m}=\left\|V_{n, m}\left(t x_{n}+(1-t) f_{1}\right)-\left(t V_{n, m} x_{n}+(1-t) V_{n, m} f_{1}\right)\right\|,
$$

then it follows from Lemma 2.4 that

$$
\begin{aligned}
b_{n, m} & \leq M_{n} g^{-1}\left(\left\|x_{n}-f_{1}\right\|-M_{n}^{-1}\left\|V_{n, m} x_{n}-V_{n, m} f_{1}\right\|\right) \\
& =M_{n} g^{-1}\left(\left\|x_{n}-f_{1}\right\|-M_{n}^{-1}\left\|x_{n+m}-f_{1}\right\|\right) .
\end{aligned}
$$

Since $\lim _{n \rightarrow \infty}\left\|x_{n}-f_{1}\right\|$ exists, fixing $m \geq 0$ and letting $n \rightarrow \infty$ in (3.1), we obtain $b_{n, m} \rightarrow 0$ as $n \rightarrow \infty$. Set $a_{n}(t)=\left\|t x_{n}+(1-t) f_{1}-f_{2}\right\|$. Then we have

$$
\begin{aligned}
a_{n+m}(t) & \leq\left\|V_{n, m}\left(t x_{n}+(1-t) f_{1}\right)-f_{2}\right\|+b_{n, m} \\
& \leq M_{n} a_{n}(t)+b_{n, m} .
\end{aligned}
$$

Fixing $n$ and then, letting $m \rightarrow \infty$ in (3.2), we get

$$
\varlimsup_{m \rightarrow \infty} a_{n+m}(t) \leq M_{n}\left[a_{n}(t)+g^{-1}\left(\left\|x_{n}-f_{1}\right\|-M_{n}^{-1} \lim _{m \rightarrow \infty}\left\|x_{n+m}-f_{1}\right\|\right)\right]
$$

and letting $n \rightarrow \infty$ in (3.3), we have

$$
\varlimsup_{n \rightarrow \infty} a_{n}(t) \leq \varliminf_{n \rightarrow \infty} a_{n}(t)+g^{-1}(0)=\varliminf_{n \rightarrow \infty} a_{n}(t) .
$$

This completes the proof.

Lemma 3.2. Let $C$ be a nonempty closed convex subset of a uniformly convex Banach space $E$. Let $T: C \rightarrow C$ be an asymptotically nonexpansive mapping. Suppose that $\left\{x_{n}\right\}$ is in $C$ such that $x_{n} \rightarrow x$ weakly and $\overline{\lim }_{m \rightarrow \infty} \overline{\lim }_{n \rightarrow \infty} \| T^{m} x_{n}-$ $x_{n} \|=0$. Then $x=T x$. 
Proof. Since $\left\{x_{n}\right\}$ converges weakly to $x \in C,\left\{x_{n}\right\}$ is a bounded sequence in $C$. Therefore, there exists $d>0$ such that $\left\{x_{n}\right\} \subset K=C \cap B_{d}$. Then $K$ is a nonempty bounded closed convex subset in $C$. Since $T: C \rightarrow C$ is asymptotically nonexpansive and thus $T: K \rightarrow C$ is also an asymptotically nonexpansive mapping. Then the rest of the proof follows as in the proof of Lemma 2.3 of Tan and $\mathrm{Xu}[11]$ and is therefore omitted.

In the sequel, let

$$
d_{n(j)}:=\frac{1}{n+1} \sum_{i=0}^{n} k_{i(j)} \quad \text { and } \quad e_{n(j)}:=\left\|\frac{1}{n+1} \sum_{i=0}^{n} T_{j}^{i} U_{n(j-1)} x_{n}-x_{n}\right\|
$$

for each $j=1,2, \ldots, r$.

Lemma 3.3. Let $E$ be a Banach space and let $C$ be a nonempty closed convex subset of $E$. Let $T_{j}: C \rightarrow C$ be an asymptotically nonexpansive mapping with sequence $\left\{k_{n(j)}\right\}_{n=0}^{\infty}$ such that $\sum_{n=0}^{\infty}\left(\frac{1}{n+1} \sum_{i=0}^{n} k_{i(j)}-1\right)_{+}<\infty$ for each $j=1,2, \ldots, r$ and $\cap_{j=1}^{r} F\left(T_{j}\right) \neq \varnothing$. Suppose that $x_{0} \in C$ and $\left\{x_{n}\right\}_{n=0}^{\infty}$ is given by (1.4). Then, $\lim _{n \rightarrow \infty}\left\|x_{n}-p\right\|$ exists for all $p \in \cap_{j=1}^{r} F\left(T_{j}\right)$.

Proof. Let $p \in \cap_{j=1}^{r} F\left(T_{j}\right)$. Then, we have

$$
\begin{aligned}
& \left\|x_{n+1}-p\right\|=\left\|U_{n(r)} x_{n}-p\right\| \\
& =\left\|\left(1-\alpha_{n(r)}\right)\left(x_{n}-p\right)+\alpha_{n(r)}\left(\frac{1}{n+1} \sum_{i=0}^{n} T_{r}^{i} U_{n(r-1)} x_{n}-p\right)\right\| \\
& \leq\left(1-\alpha_{n(r)}\right)\left\|x_{n}-p\right\|+\alpha_{n(r)} d_{n(r)}\left\|U_{n(r-1)} x_{n}-p\right\| \\
& \vdots \\
& \leq\left[1+\alpha_{n(r)}\left(d_{n(r)}-1\right)_{+}+\sum_{j=1}^{r-1}\left(\prod_{l=j}^{r} \alpha_{n(l)}\right)\left(\prod_{l=j+1}^{r} d_{n(l)}\right)\left(d_{n(j)}-1\right)_{+}\right]\left\|x_{n}-p\right\|,
\end{aligned}
$$

for all $n \in \mathbb{N}$. Since $\left(d_{n(j)}-1\right)_{+} \rightarrow 0$ as $n \rightarrow \infty$ for each $j=1,2, \ldots, r$, it implies that each $j,\left\{d_{n(j)}\right\}_{n=0}^{\infty}$ is bounded. Thus, there exists $D>0$ such that

$$
\max _{1 \leq j \leq r}\left\{\sup _{n \in \mathbb{N}}\left\{d_{n(j)}\right\}\right\} \leq D
$$

So, we have

$$
\begin{aligned}
\left\|x_{n+1}-p\right\| & \leq\left[1+\left(d_{n(r)}-1\right)_{+}+\sum_{j=1}^{r-1} D^{r-j}\left(d_{n(j)}-1\right)_{+}\right]\left\|x_{n}-p\right\| \\
& =\left(1+\varphi_{n}\right)\left\|x_{n}-p\right\|,
\end{aligned}
$$

for all $n \in \mathbb{N}$ where $\varphi_{n}:=\left(d_{n(r)}-1\right)_{+}+\sum_{j=1}^{r-1} D^{r-j}\left(d_{n(j)}-1\right)_{+}$. Since $\sum_{n=0}^{\infty}\left(d_{n(j)}-\right.$ $1)_{+}<\infty$ for each $j=1,2, \ldots, r$, we have $\sum_{n=0}^{\infty} \varphi_{n}<\infty$. Thus, $\lim _{n \rightarrow \infty}\left\|x_{n}-p\right\|$ exists by Lemma 2.1. This completes the proof of Lemma 3.3. 
Lemma 3.4. Let $C$ be a nonempty closed convex subset of a uniformly convex Banach space $E$ and let $T_{j}: C \rightarrow C$ be an asymptotically nonexpansive mapping with sequence $\left\{k_{n(j)}\right\}_{n=0}^{\infty}$ such that $\sum_{n=0}^{\infty}\left(\frac{1}{n+1} \sum_{i=0}^{n} k_{i(j)}-1\right)_{+}<\infty$ for each $j=1,2, \ldots, r$ and $\cap_{j=1}^{r} F\left(T_{j}\right) \neq \varnothing$. Let $\left\{\alpha_{n(j)}\right\}_{n=0}^{\infty}$ be a sequence in $[0,1]$ satisfying the following conditions:

$$
0<a \leq \alpha_{n(r)} \leq 1 ; \quad 0<b \leq \alpha_{n(j)} \leq c<1
$$

for all $j=1,2, \ldots, r-1$ and some constants $a, b$, and $c$.

Suppose that $x_{0} \in C$ and $\left\{x_{n}\right\}_{n=0}^{\infty}$ is given by (1.4). Then,

$$
\varlimsup_{m \rightarrow \infty} \varlimsup_{n \rightarrow \infty}\left\|T_{j}^{m} x_{n}-x_{n}\right\|=0 \quad \text { for each } j=1,2, \ldots, r .
$$

Proof. Let $p \in \cap_{j=1}^{r} F\left(T_{j}\right) \neq \varnothing$. By Lemma 3.3 and the hypotheses of Lemma 3.4 imply that $\left\{x_{n}\right\}_{n=0}^{\infty}$ and $\left\{d_{n(j)}\right\}_{n=0}^{\infty}$ are bounded for each $j=1,2, \ldots, r$. Then, there exists a constant $d>0$ such that

$$
\bigcup_{j=1}^{r}\left\{\frac{1}{n+1} \sum_{i=0}^{n} T_{j}^{n} U_{n(j-1)} x_{n}-p, U_{n(j)} x_{n}\right\}_{n=0}^{\infty} \bigcup\left\{x_{n}-p\right\}_{n=0}^{\infty} \subseteq B_{d} .
$$

By Lemma 2.5, there exists a continuous, strictly increasing and convex function $g:[0, \infty) \rightarrow[0, \infty)$ such that $g(0)=0$, and

$$
\|\lambda x+(1-\lambda) y\|^{2} \leq \lambda\|x\|^{2}+(1-\lambda)\|y\|^{2}-\lambda(1-\lambda) g(\|x-y\|)
$$

for all $x, y \in B_{d}$ and $\lambda \in[0,1]$. Using (3.7), we have

$$
\begin{aligned}
& \left\|x_{n+1}-p\right\|^{2}=\left\|U_{n(r)} x_{n}-p\right\|^{2} \\
& =\left\|\left(1-\alpha_{n(r)}\right)\left(x_{n}-p\right)+\alpha_{n(r)}\left(\frac{1}{n+1} \sum_{i=0}^{n} T_{r}^{i} U_{n(r-1)} x_{n}-p\right)\right\|^{2} \\
& \leq\left(1-\alpha_{n(r)}\right)\left\|x_{n}-p\right\|^{2}+\alpha_{n(r)}\left\|\frac{1}{n+1} \sum_{i=0}^{n} T_{r}^{i} U_{n(r-1)} x_{n}-p\right\|^{2} \\
& \quad-\alpha_{n(r)}\left(1-\alpha_{n(r)}\right) g\left(\left\|\frac{1}{n+1} \sum_{i=0}^{n} T_{r}^{i} U_{n(r-1)} x_{n}-x_{n}\right\|\right) \\
& \leq\left(1-\alpha_{n(r)}\right)\left\|x_{n}-p\right\|^{2}+\alpha_{n(r)} d_{n(r)}^{2}\left\|U_{n(r-1)} x_{n}-p\right\|^{2}-\alpha_{n(r)}\left(1-\alpha_{n(r)}\right) g\left(e_{n(r)}\right) \\
& \vdots \\
& \leq \\
& \quad+x_{n}-p \|^{2} \\
& \quad\left[\alpha_{n(r)}\left(d_{n(r)}^{2}-1\right)_{+}+\sum_{j=1}^{r-1}\left(\prod_{l=j}^{r} \alpha_{n(l)}\right)\left(\prod_{l=j+1}^{r} d_{n(l)}^{2}\right)\left(d_{n(j)}^{2}-1\right)_{+}\right]\left\|x_{n}-p\right\|^{2} \\
& \quad-\left[\alpha_{n(r)}\left(1-\alpha_{n(r)}\right) g\left(e_{n(r)}\right)+\sum_{j=1}^{r-1}\left(\prod_{l=j}^{r} \alpha_{n(l)}\right)\left(\prod_{l=j+1}^{r} d_{n(l)}^{2}\right)\left(1-\alpha_{n(j)}\right) g\left(e_{n(j)}\right)\right]
\end{aligned}
$$


for all $n=0,1,2, \ldots$. Since each $j,\left(d_{n(j)}-1\right)_{+} \rightarrow 0$ as $n \rightarrow \infty$, it implies that $\left\{d_{n(j)}\right\}_{n=0}^{\infty}$ is bounded and there exists a positive integer $N_{j}$ such that $d_{n(j)}^{2} \geq \frac{1}{2}$ for all $n \geq N_{j}$. Now, put

$$
M:=\max _{1 \leq j \leq r}\left\{\sup _{n \in \mathbb{N}}\left\{d_{n(j)}+1, d_{n(j)}^{2}\right\}\right\}<\infty
$$

and

$$
D:=\sup _{n \in \mathbb{N}}\left\{\left\|x_{n}-p\right\|\right\}<\infty .
$$

Then, by (3.8), we have

$$
\begin{aligned}
\left\|x_{n+1}-p\right\|^{2} \leq & \left\|x_{n}-p\right\|^{2}+\left[M\left(d_{n(r)}-1\right)_{+}+\sum_{j=1}^{r-1} M^{r-j+1}\left(d_{n(j)}-1\right)_{+}\right] D \\
& -\left[a\left(1-\alpha_{n(r)}\right) g\left(e_{n(r)}\right)+a(1-c) \sum_{j=1}^{r-1}\left(b^{r-j}\right)\left(\frac{1}{2}\right)^{r-j} g\left(e_{n(j)}\right)\right]
\end{aligned}
$$

for all $n \geq N$ where $N:=\max _{1 \leq j \leq r}\left\{N_{j}\right\}$. Hence by Lemma 3.3 and $\left(d_{n(j)}-\right.$ $1)_{+} \rightarrow 0$ as $n \rightarrow \infty$ for each $j=1,2, \ldots, r$, we have

$$
\begin{aligned}
& {\left[a\left(1-\alpha_{n(r)}\right) g\left(e_{n(r)}\right)+a(1-c) \sum_{j=1}^{r-1}\left(b^{r-j}\right)\left(\frac{1}{2}\right)^{r-j} g\left(e_{n(j)}\right)\right] } \\
\leq & \left\|x_{n}-p\right\|^{2}-\left\|x_{n+1}-p\right\|^{2}+\left[M\left(d_{n(r)}-1\right)_{+}+\sum_{j=1}^{r-1} M^{r-j+1}\left(d_{n(j)}-1\right)_{+}\right] D \rightarrow 0
\end{aligned}
$$

as $n \rightarrow \infty$. Thus,

$$
\left(1-\alpha_{n(r)}\right) g\left(e_{n(r)}\right) \rightarrow 0 \quad \text { as } \quad n \rightarrow \infty
$$

and

$$
g\left(e_{n(j)}\right) \rightarrow 0 \quad \text { as } \quad n \rightarrow \infty \quad \text { for each } i=1,2, \ldots, r-1 .
$$

Since $g$ is a continuous and strictly increasing function with $g(0)=0$, we have

$$
\lim _{n \rightarrow \infty}\left(1-\alpha_{n(r)}\right)\left\|\frac{1}{n+1} \sum_{i=0}^{n} T_{j}^{i} U_{n(r-1)} x_{n}-x_{n}\right\|=\lim _{n \rightarrow \infty}\left(1-\alpha_{n(r)}\right) e_{n(r)}=0
$$

and

$$
\lim _{n \rightarrow \infty}\left\|\frac{1}{n+1} \sum_{i=0}^{n} T_{j}^{i} U_{n(j-1)} x_{n}-x_{n}\right\|=\lim _{n \rightarrow \infty} e_{n(j)}=0
$$

for each $j=1,2, \ldots, r-1$.

Observe that for all $m=1,2, \ldots$

$$
\left\|T_{r}^{m} x_{n+1}-x_{n+1}\right\| \leq\left\|T_{r}^{m} x_{n+1}-T_{r}^{m}\left(\frac{1}{n+1} \sum_{i=0}^{n} T_{r}^{i} U_{n(r-1)} x_{n}\right)\right\|
$$




$$
\begin{aligned}
& +\left\|T_{r}^{m}\left(\frac{1}{n+1} \sum_{i=0}^{n} T_{r}^{i} U_{n(r-1)} x_{n}\right)-\frac{1}{n+1} \sum_{i=0}^{n} T_{r}^{i} U_{n(r-1)} x_{n}\right\| \\
& +\left\|\frac{1}{n+1} \sum_{i=0}^{n} T_{r}^{i} U_{n(r-1)} x_{n}-x_{n+1}\right\| \\
& \leq\left(k_{m(r)}+1\right)\left\|x_{n+1}-\frac{1}{n+1} \sum_{i=0}^{n} T_{r}^{i} U_{n(r-1)} x_{n}\right\| \\
& +\left\|T_{r}^{m}\left(\frac{1}{n+1} \sum_{i=0}^{n} T_{r}^{i} U_{n(r-1)} x_{n}\right)-\frac{1}{n+1} \sum_{i=0}^{n} T_{r}^{i} U_{n(r-1)} x_{n}\right\| \\
& =\left(k_{m(r)}+1\right)\left(1-\alpha_{n(r)}\right)\left\|x_{n}-\frac{1}{n+1} \sum_{i=0}^{n} T_{r}^{i} U_{n(r-1)} x_{n}\right\| \\
& +\left\|T_{r}^{m}\left(\frac{1}{n+1} \sum_{i=0}^{n} T_{r}^{i} U_{n(r-1)} x_{n}\right)-\frac{1}{n+1} \sum_{i=0}^{n} T_{r}^{i} U_{n(r-1)} x_{n}\right\|
\end{aligned}
$$

and

$$
\begin{aligned}
\left\|T_{j}^{m} x_{n}-x_{n}\right\| \leq & \left\|T_{j}^{m} x_{n}-T_{j}^{m}\left(\frac{1}{n+1} \sum_{i=0}^{n} T_{j}^{i} U_{n(j-1)} x_{n}\right)\right\| \\
& +\left\|T_{j}^{m}\left(\frac{1}{n+1} \sum_{i=0}^{n} T_{j}^{i} U_{n(j-1)} x_{n}\right)-\frac{1}{n+1} \sum_{i=0}^{n} T_{j}^{i} U_{n(j-1)} x_{n}\right\| \\
& +\left\|\frac{1}{n+1} \sum_{i=0}^{n} T_{j}^{i} U_{n(j-1)} x_{n}-x_{n}\right\| \\
\leq & \left(k_{m(j)}+1\right)\left\|x_{n}-\frac{1}{n+1} \sum_{i=0}^{n} T_{j}^{i} U_{n(j-1)} x_{n}\right\| \\
& +\left\|T_{j}^{m}\left(\frac{1}{n+1} \sum_{i=0}^{n} T_{j}^{i} U_{n(j-1)} x_{n}\right)-\frac{1}{n+1} \sum_{i=0}^{n} T_{j}^{i} U_{n(j-1)} x_{n}\right\|
\end{aligned}
$$

for each $j=1,2, \ldots, r-1$. Hence, by (3.10)-(3.13) and Lemma 2.2, we have

$$
\varlimsup_{m \rightarrow \infty} \varlimsup_{n \rightarrow \infty}\left\|T_{j}^{m} x_{n}-x_{n}\right\|=0
$$

for each $j=1,2, \ldots, r$. This completes the proof of Lemma 3.4.

We will now prove our main theorems.

Theorem 3.5. Let $C$ be a nonempty closed convex subset of a uniformly convex Banach space $E$ which satisfies Opial's condition or its dual $E^{*}$ has the KK-property and $T_{j}: C \rightarrow C$ be an asymptotically nonexpansive mapping with sequence $\left\{k_{n(j)}\right\}_{n=0}^{\infty}$ such that $\sum_{n=0}^{\infty}\left(\frac{1}{n+1} \sum_{i=0}^{n} k_{i(j)}-1\right)_{+}<\infty$ for each $j=1,2, \ldots, r$ and $\cap_{j=1}^{r} F\left(T_{j}\right) \neq \varnothing$. Let $\left\{\alpha_{n(j)}\right\}_{n=0}^{\infty}$ be a sequence in $[0,1]$ 
satisfying the following conditions:

$$
0<a \leq \alpha_{n(r)} \leq 1 ; \quad 0<b \leq \alpha_{n(j)} \leq c<1
$$

for all $j=1,2, \ldots, r-1$ and some constants $a, b$, and $c$.

Suppose that $x_{0} \in C$ and $\left\{x_{n}\right\}_{n=0}^{\infty}$ is given by (1.4). Then, $\left\{x_{n}\right\}_{n=0}^{\infty}$ converges weakly to a common fixed point of $T_{1}, T_{2}, \ldots, T_{r}$.

Proof. Define a mapping $S_{n}: C \rightarrow C$ by $S_{n} x=U_{n(r)} x, x \in C$. Then, $x_{n+1}=$ $S_{n} x_{n}$ and $\cap_{j=1}^{r} F\left(T_{j}\right) \subseteq F\left(S_{n}\right)$. Moreover, for all $x, y \in C$, we have

$$
\begin{aligned}
\| S_{n} x & -S_{n} y\|=\| U_{n(r)} x-U_{n(r)} y \| \\
& \leq\left(1-\alpha_{n(r)}\right)\|x-y\|+\alpha_{n(r)} \frac{1}{n+1} \sum_{i=0}^{n}\left\|T_{r}^{i} U_{n(r-1)} x-T_{r}^{i} U_{n(r-1)} y\right\| \\
& \leq\left(1-\alpha_{n(r)}\right)\|x-y\|+\alpha_{n(r)} d_{n(r)}\left\|U_{n(r-1)} x-U_{n(r-1)} y\right\| \\
& \vdots \\
& \leq\left[1+\alpha_{n(r)}\left(d_{n(r)}-1\right)_{+}+\sum_{j=1}^{r-1}\left(\prod_{l=j}^{r} \alpha_{n(l)}\right)\left(\prod_{l=j+1}^{r} d_{n(l)}\right)\left(d_{n(j)}-1\right)_{+}\right]\|x-y\| \\
& \leq\left(1+\varphi_{n}\right)\|x-y\|,
\end{aligned}
$$

where $\varphi_{n}$ is as in the proof of Lemma 3.3 and $\sum_{n=0}^{\infty} \varphi_{n}<\infty$. Thus, $S_{n}$ is Lipschitzian with the Lipschitz constant $L_{n}:=1+\varphi_{n}$ such that $\sum_{n=0}^{\infty}\left(L_{n}-1\right)=$ $\sum_{n=0}^{\infty} \varphi_{n}<\infty$ and $\cap_{n=0}^{\infty} F\left(S_{n}\right) \supseteq \cap_{j=1}^{r} F\left(T_{j}\right) \neq \varnothing$.

It follows from Lemmas 3.4 and 3.2 that $\omega_{w}\left(x_{n}\right) \subseteq \cap_{j=1}^{r} F\left(T_{j}\right)$. So to show that $\left\{x_{n}\right\}$ converges weakly to a common fixed point of $T_{1}, T_{2}, \ldots, T_{r}$, it suffices to show that $\omega_{w}\left(x_{n}\right)$ consists of just one point. In case $E^{*}$ has the KK-property, it is easy to see from Lemmas 3.1 and 2.3 that the theorem is true. So, we suppose next that $E$ satisfies Opial's condition. This follows basically as in the proof of Theorem 1 of [1] using Lemmas 3.3, 3.4, and 3.2. This completes the proof of Theorem 3.5.

As a consequence of Theorem 3.5, we obtain the following result.

Theorem 3.6. Let $C$ be a nonempty closed convex subset of a uniformly convex Banach space $E$ which satisfies Opial's condition or its dual $E^{*}$ has the $K K$-property and $T_{j}: C \rightarrow C$ be nonexpansive mapping for each $j=1,2, \ldots, r$ such that $\cap_{j=1}^{r} F\left(T_{j}\right) \neq \varnothing$. Let $\left\{\alpha_{n(j)}\right\}_{n=0}^{\infty}$ be a sequence in $[0,1]$ satisfying the following conditions:

$$
0<a \leq \alpha_{n(r)} \leq 1 ; \quad 0<b \leq \alpha_{n(j)} \leq c<1
$$

for all $j=1,2, \ldots, r-1$ and some constants $a, b$, and $c$.

Suppose that $x_{0} \in C$ and $\left\{x_{n}\right\}_{n=0}^{\infty}$ is given by (1.4). Then, $\left\{x_{n}\right\}_{n=0}^{\infty}$ converges weakly to a common fixed point of $T_{1}, T_{2}, \ldots, T_{r}$. 


\section{ACKNOWLEDGEMENTS}

The author is grateful to the referees for his/her suggestions to the improvement of this paper and the Kuang Wu Institute of Technology for its generous support during the preparation of this manuscript.

This work was supported by the National Science Council of the Republic of China under Contract NSC 91-2115-M-149-001.

\section{REFERENCES}

1. S. Atsushiba and W. Takahashi, Approximating common fixed points of two nonexpansive mappings in Banach spaces. Bull. Austral. Math. Soc. 57(1998), No. 1, 117-127.

2. R. E. BRUCK, A simple proof of the mean ergodic theorem for nonlinear contractions in Banach spaces. Israel J. Math. 32(1979), No. 2-3, 107-116.

3. S. S. Chang, Y. J. Cho, and H. Zhou, Demi-closed principle and weak convergence problems for asymptotically nonexpansive mappings. J. Korean Math. Soc. 38(2001), No. 6, 1245-1260.

4. J. Diestel, Geometry of Banach spaces - selected topics. Lecture Notes in Mathematics, 485. Springer-Verlag, Berlin-New York, 1975.

5. K. FAn and I. Glicksberg, Some geometric properties of the spheres in a normed linear space. Duke Math. J. 251958 553-568.

6. J. García Falset, W. Kaczor, T. Kuczumow, and S. Reich, Weak convergence theorems for asymptotically nonexpansive mappings and semigroups. Nonlinear Anal. 43(2001), No. 3, Ser. A: Theory Methods, 377-401.

7. K. Goebel and W. A. Kirk, A fixed point theorem for asymptotically nonexpansive mappings. Proc. Amer. Math. Soc. 35(1972), 171-174.

8. K. Goebel and W. A. Kirk, Topics in metric fixed point theory. Cambridge Studies in Advanced Mathematics, 28. Cambridge University Press, Cambridge, 1990.

9. Z. OPIAL, Weak convergence of the sequence of successive approximations for nonexpansive mappings. Bull. Amer. Math. Soc. 73(1967), 591-597.

10. N. Shioji and W. TAKahashi, Strong convergence of averaged approximants for asymptotically nonexpansive mappings in Banach spaces. J. Approx. Theory 97(1999), No. 1, $53-64$.

11. K.-K. TAN and H.-K. XU, The quadratic minimum spanning tree problem. Naval Res. Logist. 39(1992), No. 3, 399-417.

12. K.-K. TAN and H.-K. XU, Fixed point iteration processes for asymptotically nonexpansive mappings. Proc. Amer. Math. Soc. 122(1994), No. 3, 733-739.

13. H. K. XU, Inequalities in Banach spaces with applications. Nonlinear Anal. 16(1991), No. $12,1127-1138$.

(Received 4.09.2003; revised 4.12.2003)

Author's address:

Center for General Education

Kuang Wu Institute of Technology

Peito, Taipei, Taiwan, 11271

R.O.C.

E-mail: juichi@kwit.edu.tw 